\title{
Categorization of Common Pigmented Skin Lesions (CPSL) using Multi-Deep Features and Support Vector Machine
}

\section{SANTI BEHERA}

Veer Surendra Sai University of Technology

PRABIRA SETHY ( $\sim$ prabirsethy.05@gmail.com )

Department of Electronics, Sambalpur University, Odisha, India

\section{Research Article}

Keywords: Common Pigmented Skin Lesions, Multi-deep features, Support vector machine, Skin cancer, Convolutional neural network

Posted Date: January 5th, 2021

DOI: https://doi.org/10.21203/rs.3.rs-136988/v1

License: (c) (i) This work is licensed under a Creative Commons Attribution 4.0 International License.

Read Full License 


\title{
Categorization of Common Pigmented Skin Lesions (CPSL) using Multi-Deep Features and Support Vector Machine
}

\author{
Prabira Kumar Sethy ${ }^{1,}$, Santi Kumari Behera ${ }^{2}$ \\ ${ }^{1}$ Department of Electronics, Sambalpur University, Odisha, India \\ ${ }^{2}$ Department of Computer Science and Engineering, Veer Surendra Sai University of \\ Technology, Odisha, India \\ *Correspondence: prabirasethy.05@gmail.com, Tel.: +91-9439489214
}

\begin{abstract}
The skin is the main organ. It is approximately 8 pounds for the average adult. Our skin is a truly wonderful organ. It isolates us and shields our bodies from hazards. However, the skin is also vulnerable to damage and distracted from its original appearance; brown, black, or blue, or combinations of those colors, known as pigmented skin lesions. These common pigmented skin lesions (CPSL) are the leading factor of skin cancer, or can say these are the primary causes of skin cancer. In the healthcare sector, the categorization of CPSL is the main problem because of inaccurate outputs, overfitting, and higher computational costs. Hence, we proposed a classification model based on multi-deep feature and support vector machine (SVM) for the classification of CPSL. The proposed system comprises two phases: first, evaluate the $11 \mathrm{CNN}$ model's performance in the deep feature extraction approach with SVM. Then, concatenate the top performed three CNN model's deep features and with the help of SVM to categorize the CPSL. In the second step, 8192 and 12288 features are obtained by combining binary and triple networks of 4096 features from the top performed CNN model. These features are also given to the SVM classifiers. The SVM results are also evaluated with principal component analysis (PCA) algorithm to the combined feature of 8192 and 12288 . The highest results are obtained with 12288 features. The experimentation results, the combination of the deep feature of Alexnet, VGG16 \& VGG19, achieved the highest accuracy of $91.7 \%$ using SVM classifier. As a result, the results show that the proposed methods are a useful tool for CPSL classification.
\end{abstract}

Keywords: Common Pigmented Skin Lesions; Multi-deep features; Support vector machine; Skin cancer; Convolutional neural network. 


\section{Introduction}

The largest organ in the human body is the skin. Various forms of inflammatory, bacterial, and viral infections spread throughout the skin, leading to several skin diseases and health problems [1]. "Melanoma is the most dangerous type of skin cancer, which has been one of the most important challenges of public health in recent years [2]."

According to the latest statistics, in 2018, 91270 new melanoma cases are diagnosed and treated in the United States [1]. "Melanoma incidence rates and mortality from this disease are projected to increase over the next few decades [3]." "The latest study shows that the diagnosis of new melanoma cases has risen by 53\% annually from 2008 to 2018[1, 4]." If it is possible to detect this type of cancer at its early stage by selecting the right treatment $[5,6]$, survival rates are very promising [7]. Otherwise, a patient's estimated 5-year survival rate would be decreased from $99 \%$ to $14 \%$ [8-10]. There was also a drastic rise in the diagnosis of new cases between 1994 and 2014, up to 77\%, in the non-melanoma form of cancer. "The most common form of non-melanoma skin cancer is basal cell carcinoma, resulting in 3000 deaths per year [11]." Therefore, this will give rise to a strong demand for primary inspection and identification of various skin cancer forms to prevent it from getting worse and provide a better prognosis [3]. The normal way to diagnose melanoma is by examining the lowprecision visual information [12,13]. "Dermoscopy, on the other hand, is a non-invasive technique that can capture a high-resolution skin image that helps dermatologists to identify features invisible to the naked eye [14]."

Several meta-analyses have shown that melanoma diagnosis is improved and reliable in naked eye examination using dermoscopy [15-17]. Even for experienced clinicians, the manual approach is challenging and subjective [18, 22-23]. Due to the similarity of visual characteristics between malignant tumors of the skin and benign lesions, dermatologists face difficulty distinguishing between them. Even if highly trained dermatologists and physicians have the average sensitivity for recognition of melanoma, it is less than $80 \%$ [24, 25]. Again, highly trained dermatologists are not available all over the world. Therefore, automatic skin lesion classification at the same level and accuracy as dermatologists or even higher is crucial in public health.

To solve the aforementioned issues, there have been plenty of publications on exploring methods to classify skin cancers by means of computer assistance. These algorithms use various approaches to the segmentation, detection, and classification of melanoma by 
integrating areas such as image processing, computer vision, and machine learning [20, 2629]. "The main problem of this type of scientific paper is the scarcity of data. However, skin cancer has been categorized into different stages according to 'man-made' methods, which is a major flaw in the classification method [30-33]."

In most current studies, the three main skin disorders, including seborrheic keratosis (SK), squamous cell (SC), and melanoma, are considered. In the last few decades, several researchers have developed various approaches to diagnose skin diseases. "In addition, natural computing system, region of interest (ROI), gray level co-occurrence matrix (GLCM), fractal-based regional texture analysis (FRTA) feature extraction methods, various machine learning algorithms such as Artificial Neural Network (ANN), Convolutional Neural Network (CNN), Generative Adversarial Networks (GANs) with Support Vector Machine (SVM), Naive Bayes were implemented [34-36]." These approaches have produced improved classification accuracy in terms of skin disease detection. Still, they have few deficiencies in higher dimensional space computational complexity, more processing time, overfitting problems, a noisy environment, and so on. To overcome this, we have proposed a classification model that uses multi-deep features and SVM to categorize CPSL. The main contribution of the article is summarized as follows.

- The dataset images were collected from the HAM-10000 dataset belongs to seven classes of CPSL such as melanoma (mel), melanocytic nevi (nv), basal cell carcinoma (bcc), actinic keratosis and carcinoma benign keratosis (bkl), dermato fibroma (df) and vascular lesions (vasc).

- The performance of $11 \mathrm{CNN}$ models is evaluated to classify seven CPSL based on their deep feature and SVM.

- High-dimensional features such as 8192 and 12288 are obtained by combining binary and triplet networks of three top-performing CNN architectures and feed to the SVM for classification.

- The performance is also evaluated by introducing dimensional reduction techniques like PCA.

- The LR+ is 49 , which is greater than 10 , and implicates the proposed method has a significant contribution to the diagnosis and an excellent diagnostic test.

- Finally, the AUC value of the proposed method is 0.99 , which is within the range of 0.9 to 1 . It reveals an excellent diagnostic approach as per STARD (Standards for Reporting of Diagnostic Accuracy Studies) guidelines. 
The remaining of the manuscript is organized as follows. Section 2 discusses the related works. Section 3 detailed the dataset. The methodology is presented in section 4 . Experimental results of the proposed method are given in Section 5. Finally, section 6 shows the discussion and conclusion section where the results are discussed by comparing with the previous methods.

\section{Related Works}

Many approaches are reported towards the classification of different skin cancer lesions or CPSL, especially in machine learning. The integrated computer-aided mechanism [35] was proposed for the detection of skin diseases. They analyze the shape, color, texture, and irregular border of the skin lesion to create various quantitative features before classification. Textural information is quantified through a fractal-based regional texture analysis (FRTA) and a gray-level co-occurrence matrix (GLCM). The radial base function (RBF) and the support vector machine (SVM) evaluate the classification's efficiency. The experimental study delivers $99.65 \%, 97.54 \%$, and $98.99 \%$ for BCC, dysplastic nevi, and melanoma classification, but the characteristic dimensional space is higher. The computer-aided classification system [36] was implemented to diagnose suspected pigmented lesions. Preselected single lesion images train computer-aided diagnostic tools for skin lesions. One hundred thirty-three patients with a multitude of skin lesions were selected for this study. The board-certified dermatologist investigates all lesions that are classified as normal or abnormal. The sensitivity of 100 percent of suspected pigmented lesions is achieved on the basis of the test set. At the population level, this computer-aided classification method is very useful for skin screening.

Dynamic Graphics Cut algorithm (DGCA) and Naive Bayes (NB), a classifier is proposed for the segmentation and classification of skin diseases [37]. Experimental datasets are taken from the International Skin Imaging Partnership (ISIC 207) [44], which shows better results compared to state-of-the-art approaches such as SegNet and FCN. Here, there are three kinds of cancers, such as keratosis, melanoma, and benign cases. The detailed analysis reveals $92.9 \%, 91.2 \%$, and $94.3 \%$ for keratosis, melanoma, and benign disease classification. It was proposed that the deep convolutional neural network (DCNN) classifier should be used to identify skin diseases [38]. DenseNet-201, InceptionResNet-v2, ResNet-50 and Inception-v3 are used in the convolutional neural network classifier. The full resolution convolutional 
network (FrCN) segments the skin lesion boundary of the entire dermoscopy image. The ISIC 2016 [27], ISIC 2017 [44], and ISIC 2018 [45] datasets are used to evaluate the performance of convolutional neural network classifiers in the case of skin disease classification.

Multi-label classification techniques are proposed [39] to identify skin disorders such as SK, BCC, nevus, and melanoma. Cross-spectrum and cross-correlation approaches for the extraction of features have been adopted. The extraction of both spectral and spatial features is clarified by suitable kernel patches and related visual impacts. Classifications of epidermal, benign melanocytic, and benign melanocytic skin lesions are performed using a multi-label multi-class skin lesion classification mechanism. Generative adversarial networks (GANs) have been developed to identify skin lesions [40]. The GAN-based data increase process improves the efficiency of the skin lesion classification. High-quality skin lesion images are synthesized by changing both the discriminator and the generator. Quantitative assessment measures such as Recall, Accuracy, Inception Distance (FID), and Inception Score (IS) assess GAN-based skin lesion's efficiency. The experimental analysis, 96.6\% average precision, $74.3 \%$ specificity, $83.2 \%$ sensitivity, and $95.2 \%$ accuracy are obtained. Present methodologies used in the classification of skin diseases are illustrated in Table 1.

Table 1. A literature review of CPSL classification

\begin{tabular}{|c|c|c|c|c|c|}
\hline $\begin{array}{l}\text { Referenc } \\
\text { es }\end{array}$ & Methods & $\begin{array}{l}\text { Name of } \\
\text { the dataset }\end{array}$ & Advantages & Limitations & Accuracy \\
\hline$[35]$ & $\begin{array}{l}\text { RBF and } \\
\text { SVM }\end{array}$ & $\begin{array}{l}\text { Real-time } \\
\text { dataset }\end{array}$ & $\begin{array}{l}\text { The higher degree of } \\
\text { accuracy and sensitivity at } \\
\text { every stage }\end{array}$ & $\begin{array}{l}\text { Higher feature } \\
\text { dimensionality } \\
\text { space }\end{array}$ & $98.79 \%$ \\
\hline [36] & $\begin{array}{l}\text { Computer- } \\
\text { aided } \\
\text { classificatio } \\
\mathrm{n}\end{array}$ & $\begin{array}{l}\text { Dermato } \\
\text { logical } 1 \\
\text { database } \\
\text { s }\end{array}$ & $\begin{array}{l}\text { Low feature dimensionality } \\
\text { with the fast-screening } \\
\text { process }\end{array}$ & $\begin{array}{l}\text { Lower } \\
\text { classification } \\
\text { accuracy }\end{array}$ & $75.9 \%$ \\
\hline [37] & $\begin{array}{l}\text { DGCA and } \\
\text { NB }\end{array}$ & ISIC 2017 & $\begin{array}{l}\text { Easy to implement the } \\
\text { classifier with } \\
\text { quick output prediction }\end{array}$ & $\begin{array}{l}\text { Higher cost and } \\
\text { complexity }\end{array}$ & $94.3 \%$ \\
\hline [38] & DCNN & $\begin{array}{l}\text { ISIC 2016, } \\
\text { ISIC } \\
2017 \text {, and } \\
\text { ISIC 2018 } \\
\text { datasets }\end{array}$ & $\begin{array}{l}\text { Better classification } \\
\text { performances } \\
\text { with accurate results }\end{array}$ & $\begin{array}{l}\text { The limited size } \\
\text { of deep learning } \\
\text { networks for } \\
\text { both training } \\
\text { and testing }\end{array}$ & $98.79 \%$ \\
\hline [39] & $\begin{array}{l}\text { Multi-label } \\
\text { classification } \\
\text { techniques }\end{array}$ & $\begin{array}{l}\text { Real-time } \\
\text { database }\end{array}$ & $\begin{array}{l}\text { Malignant and benign } \\
\text { lesions in the classes of } \\
\text { melanoma, nevus, } \\
\text { BCC and SK have been } \\
\text { identified. }\end{array}$ & $\begin{array}{l}\text { It takes more } \\
\text { processing time. }\end{array}$ & $98.79 \%$ \\
\hline
\end{tabular}




\section{About Dataset}

We considered the image classification task of the most common lesions that should be distinguished in a skin cancer screening setting. About $90 \%$ of lesions found in the routine skin examination are covered by the following seven skin lesion classes: (1) Actinic keratoses and intraepithelial carcinoma / Bowen's disease (akiec), (2) basal cell carcinoma (bcc), (3) benign keratosis-like lesions (solar lentigines / seborrheic keratoses and lichenplanus like keratoses, (bkl), (4) dermatofibroma (df), (5) melanoma (mel), (6) melanocytic nevi (nv), and (7) vascular lesions (angiomas, angiokeratomas, pyogenic granulomas and hemorrhage (vasc). All images were obtained from the HAM10000 Dataset [41]. This dataset contains 10015 dermatoscopic images, which can serve as a training set for academic machine learning purposes. There are different dermatoscopy dataset publicly available, illustrated in Table 2. In this study, the HAM1000 dataset is taken into consideration because: (1) the only dataset which contains seven kinds of CPSL, (2) all seven kinds of CPSL have more than 100 images, (3) 53.3\% of images are pathologically verified.

Table 2. Summary of publicly available dermatoscopic image datasets in comparison to HAM10000

\begin{tabular}{l|l|l|l|l|l|l|l|l|c}
\hline Dataset & $\begin{array}{c}\text { Total } \\
\text { images }\end{array}$ & $\begin{array}{c}\text { Pathologic } \\
\text { verification (\%) }\end{array}$ & akiec & bcc & bkl & df & mel & nv & vasc \\
\hline PH2 & 200 & $20.5 \%$ & - & - & - & - & 40 & 160 & - \\
\hline Atlas & 1024 & unknown & 5 & 42 & 70 & 20 & 275 & 582 & 30 \\
\hline ISIC 2017 & 13786 & $26.3 \%$ & 2 & 33 & 575 & 7 & 1019 & 11861 & 15 \\
\hline Rosendahl & 2259 & $100 \%$ & 295 & 296 & 490 & 30 & 342 & 803 & 3 \\
\hline ViDIR Legacy & 439 & $100 \%$ & 0 & 5 & 10 & 4 & 67 & 350 & 3 \\
\hline ViDIR Current & 3363 & $77.1 \%$ & 32 & 211 & 475 & 51 & 680 & 1832 & 82 \\
\hline ViDIR MoleMax & 3954 & $1.2 \%$ & 0 & 2 & 124 & 30 & 24 & 3720 & 54 \\
\hline HAM10000 & 10015 & $53.3 \%$ & 327 & 514 & 1099 & 115 & 1113 & 6705 & 142 \\
\hline
\end{tabular}

To make the dataset balanced across all classes, we have horizontally and vertically flipped the images of df, akiec, and vasc class; and chosen a subset of images from bkl, mel, and nv class. The dataset distribution of CPSL is taken in this research is given in Table 3. Figure 1 illustrates the sample images of seven kinds of CPSL.

Table 3. Distribution of Sample images of CPSL

\begin{tabular}{|l|l|l|l|l|l|l|l|}
\hline akiec & bcc & bkl & df & mel & nv & vasc & Total \\
\hline
\end{tabular}




\begin{tabular}{l|l|l|l|l|l|l|l}
\hline 500 & 514 & 500 & 345 & 500 & 500 & 426 & 3285 \\
\hline
\end{tabular}

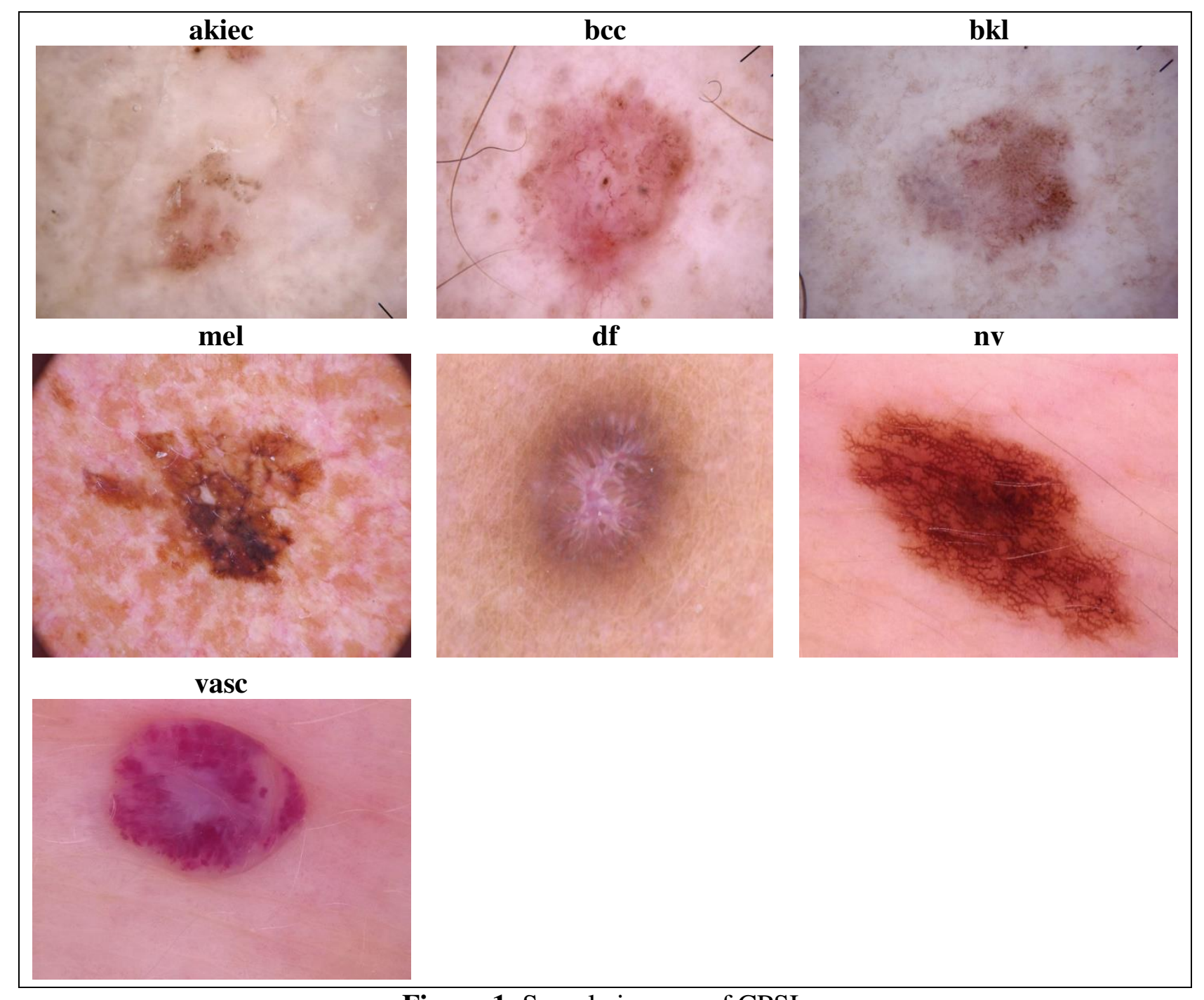

Figure 1. Sample images of CPSL

\section{Methodology}

The proposed method comprises two phases: first, the 11CNN models are evaluated for classification of CPSL based on the feature extraction approach. Here, the features are extracted from fully connected layer and feed to the SVM for classification. The linear SVM is used for classification. When SVM is used as a classifier in CNN models, it increases accuracy $[42,43]$. The performance evaluation model of CNN for the classification of CPSL is shown in Figure 2. In the second phase, the features taken from three top performed models are combined and applied to the same SVM classifiers. In the last step of the experiment, 
features are given to the classifiers after PCA. The architectural design of the proposed method is shown in Figure 3. The strategy for evaluating all classification models in this study is based on a training and testing ratio of 80:20.

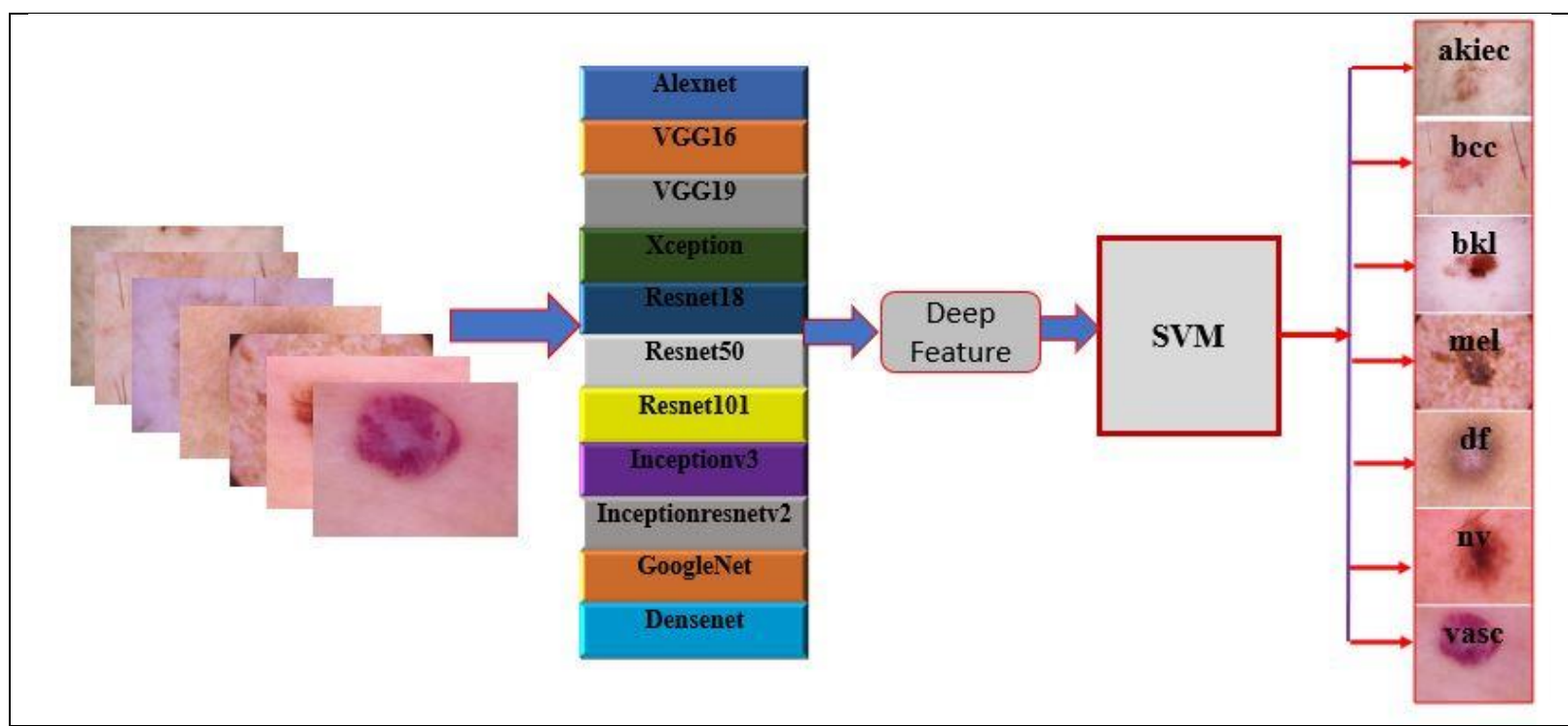

Figure 2. Performance Evaluation model of CNN for classification of CPSL

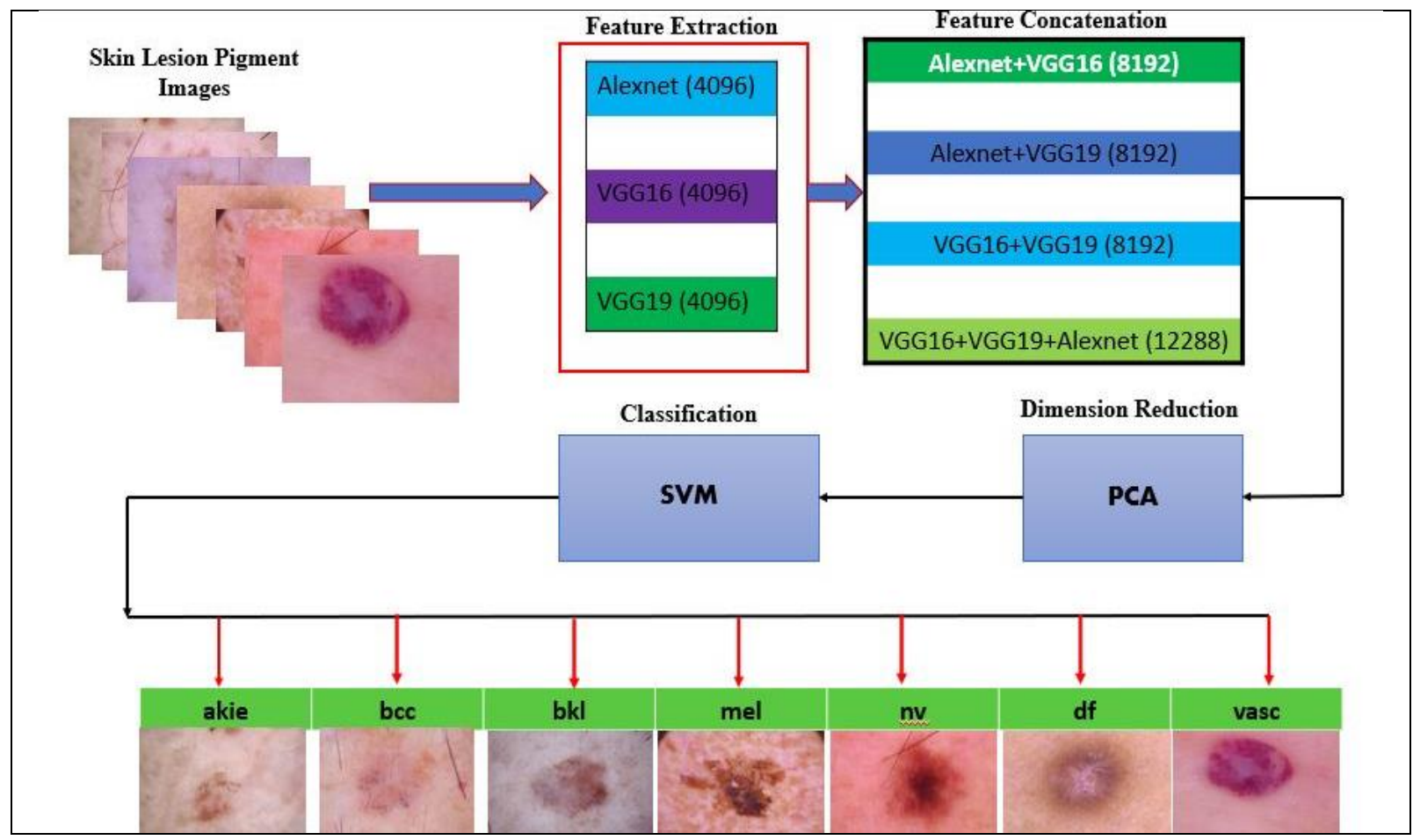

Figure 3. The framework of the Proposed method based on multi-deep Features and SVM for the classification of CPSL

\section{Experimental Analysis and Results}


Evaluating a method is important in building a useful machine learning model. There are many performance criteria used to test the performance of the classification. The most commonly used classification evaluation metric is "accuracy." However, this criterion alone is not always correct and can be misleading in some cases. Therefore, it will be useful to provide an evaluation by looking at other criteria that support accuracy. The metrics we use to choose the top performed CNN model in this study; are accuracy, sensitivity, specificity, precision, FPR, and F1 score.

Table 4. Performance evaluation parameters computed using the deep feature of the CNN model and SVM

\begin{tabular}{|c|c|c|c|c|c|c|}
\hline Model & Accuracy & Sensitivity & Specificity & Precision & FPR & F1 Score \\
\hline Alexnet & 0.717142857 & 0.717142857 & 0.952857143 & 0.771124988 & 0.047142857 & 0.713513735 \\
\hline VGG16 & 0.723095238 & 0.723095238 & 0.953849206 & 0.75281268 & 0.046150794 & 0.714124799 \\
\hline VGG19 & 0.735714286 & 0.735714286 & 0.955952381 & 0.749490933 & 0.044047619 & 0.732478894 \\
\hline Xception & 0.584047619 & 0.584047619 & 0.930674603 & 0.652361318 & 0.069325397 & 0.568450918 \\
\hline Resnet18 & 0.428809524 & 0.428809524 & 0.904801587 & 0.648393615 & 0.095198413 & 0.394574751 \\
\hline Resnet50 & 0.58047619 & 0.58047619 & 0.930079365 & 0.705257431 & 0.069920635 & 0.576618549 \\
\hline Resnet101 & 0.677380952 & 0.677380952 & 0.946230159 & 0.730705187 & 0.053769841 & 0.678646795 \\
\hline Inceptionv3 & 0.613095238 & 0.613095238 & 0.935515873 & 0.679088471 & 0.064484127 & 0.603739113 \\
\hline $\begin{array}{l}\text { Inceptionresnet } \\
\text { v2 }\end{array}$ & 0.565 & 0.565 & 0.9275 & 0.636205758 & 0.0725 & 0.565969183 \\
\hline GoogleNet & 0.525952381 & 0.525952381 & 0.920992063 & 0.622583676 & 0.079007937 & 0.526559175 \\
\hline Densenet & 0.655952381 & 0.655952381 & 0.94265873 & 0.695805106 & 0.05734127 & 0.654849059 \\
\hline
\end{tabular}

It was observed from Table 4, the Alexnet, VGG16, and VGG19 are the three top performed CNN model for classification of CPSL. The deep features of these three top performed models are now combined and feed to SVM for classification. The results are recorded in Table 5 in terms of Accuracy and AUC. The classification model is also evaluated with the employment of PCA, and corresponding results are recorded in Table 6.

Table 5. Performance of classification models with combined deep features

\begin{tabular}{l|l|l|l|l}
\hline Models & Features & Accuracy $(\%)$ & AUC & $\begin{array}{l}\text { Total } \\
\text { misclassification }\end{array}$ \\
\hline
\end{tabular}




\begin{tabular}{l|l|l|l|l}
\hline Alexnet+ VGG16 & 8192 & 79.4 & 0.89 & 138 \\
\hline Alexnet+VGG19 & 8192 & 78.9 & 0.92 & 127 \\
\hline VGG16+VGG19 & 8192 & 82.5 & 0.95 & 102 \\
\hline Alexnet+vgg16+vgg19 & 12288 & 91.7 & 0.99 & 84 \\
\hline
\end{tabular}

Table 6. Performance of classification models with combined deep features and PCA

\begin{tabular}{ll|l|l|l}
\hline Models & Features & Accuracy $(\%)$ & AUC & $\begin{array}{l}\text { Total } \\
\text { misclassification }\end{array}$ \\
\hline Alexnet+ VGG16 & 8192 & 78.9 & 0.88 & 158 \\
\hline Alexnet+VGG19 & 8192 & 77.5 & 0.91 & 134 \\
\hline VGG16+VGG19 & 8192 & 79.9 & 0.95 & 112 \\
\hline Alexnet+vgg16+vgg19 & 12288 & 85.5 & 0.99 & 92 \\
\hline
\end{tabular}

It was observed from Table 5 and Table 6; the performance of classification models is degraded with the employment of PCA. In the combination of deep features of binary and triplet CNN architectures, the performance of classification models is increased compared to an individual. Among the combined deep feature, the effectiveness of the deep features of triplet CNN architecture is more than the deep feature of binary CNN architectures. The confusion matrix and ROC plot of classification models, i.e., based on triplet deep feature and SVM, are presented in Figures 4 and 5, respectively. 


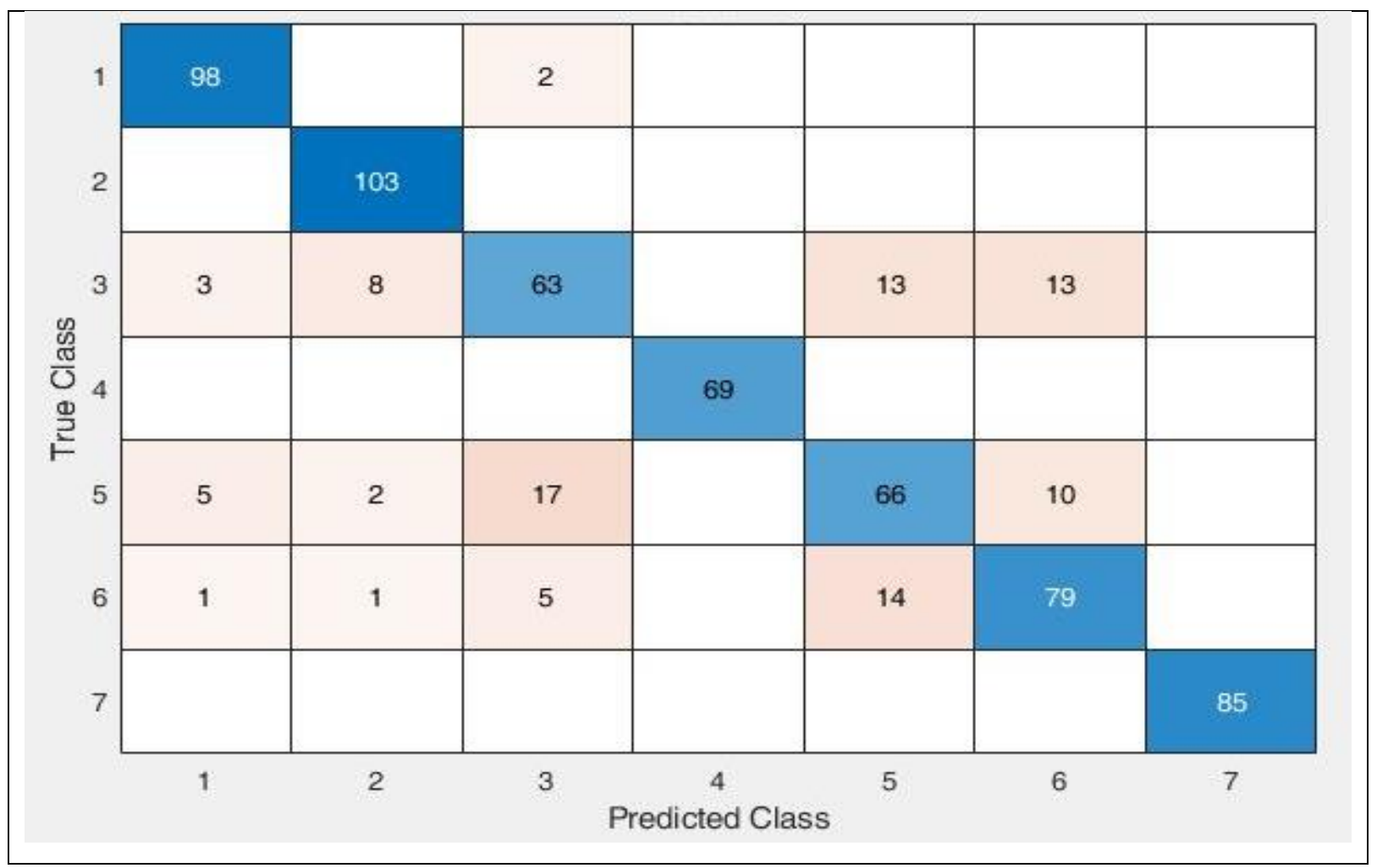

Figure 4. Confusion Matrix of Proposed classification model for diagnosis of breast carcinoma

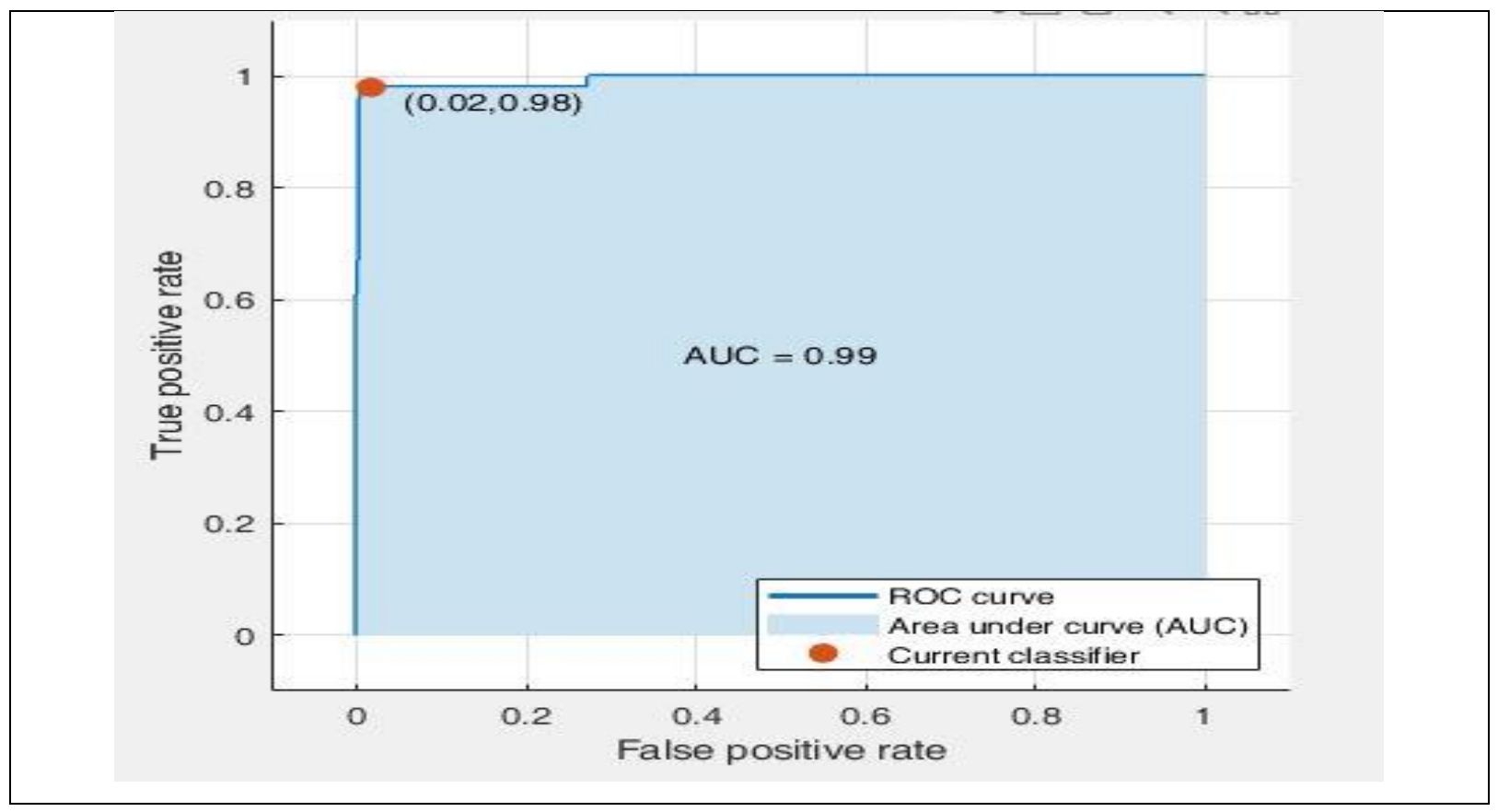

Figure 5. AUC of Proposed classification model for diagnosis of breast carcinoma

Figure 5 shows that the true positive rate (TPR) and false-positive rate (FPR) of the classification model are 0.02 and 0.98 , respectively. The positive likelihood ratio (LR+) is calculated by equation 1 .

$$
L R+=\frac{T P R}{F P R}
$$


Hence, for proposed classification model $L R+=\frac{0.98}{0.02}=49$. So, as per STARD [46], the proposed method is a good diagnostic test. In addition, the AUC value of the proposed method is 0.99 , which is within the range of 0.9 to 1.0 . It reveals an excellent diagnostic approach as per STARD (Standards for Reporting of Diagnostic Accuracy Studies) guidelines [46]. Further, Table 7 presents the true positive rates (TPR) and positive predictive values (PPV) value of seven classes of CPSL.

Table 7. TPR and PPV values of each CPSL classes

\begin{tabular}{l|l|l}
\hline Classes & TPR $(\%)$ & PPV $(\%$ \\
\hline akiec & 98 & 91.6 \\
\hline bcc & 100 & 90.4 \\
\hline bkl & 63.0 & 72.4 \\
\hline mel & 100 & 100 \\
\hline nv & 66 & 71.0 \\
\hline df & 79 & 77.5 \\
\hline vasc & 100 & 100 \\
\hline
\end{tabular}

It was observed from Table 7 , the TPR or sensitivity of akiec is $98 \%$, bcc is $100 \%$, bkl is $63 \%$, mel is $100 \%$, nv is $66 \%$, df is $79 \%$, and vasc is $100 \%$. The TPR values of classes are more than $60 \%$, and the PPV values are more than $70 \%$. Hence, it indicates the proposed methodology is going on a positive approach towards the classification of CPSL.

\section{Conclusion}

Our study aims to increase the classification accuracy values of the applied dataset with the models obtained by using two different forms of deep feature extraction approach with SVM. In the first, approach the deep features of $11 \mathrm{CNN}$ models with SVM are evaluated for classification of CPSL. Then, the three top performed CNN architectures are chosen to prepared large dimensional deep features. The 8192 and 12288 dimensional deep features are prepared by combining binary and triplet network. Then, these features are fed to the SVM for classification of seven kinds of CPSL. Further, PCA is also employed to evaluate those classification model. It reveals from the experimental results that the 12288-dimensional deep feature with SVM performed well to classify seven kinds of CPSL. The achieved accuracy and AUC are $91.7 \%$ and 0.99 , respectively, higher than the state-of-art.

Further, no research paper is published to classify seven kinds of CPSL, illustrated in Table 8. 
Table 8. Performance Comparison of the proposed method with state-of-the-art techniques

\begin{tabular}{|c|c|c|c|}
\hline Work & Database & CPSL Classes & Classification Performance \\
\hline Oliveira et al. [46] & ISIC: dataset & $\begin{array}{l}\text { Malignant and } \\
\text { benign }\end{array}$ & $\begin{array}{l}\text { Accuracy-94.3\% } \\
\text { Sensitivity-91.8\% } \\
\text { Specificity-96.7\% }\end{array}$ \\
\hline Kasmi et al. [47] & $\begin{array}{l}\text { EDRA, Interactive Atlas } \\
\text { of Dermoscopy }\end{array}$ & $\begin{array}{l}\text { Malignant and } \\
\text { benign }\end{array}$ & $\begin{array}{l}\text { Sensitivity }-91.25 \% \\
\text { Specificity }-95.83 \%\end{array}$ \\
\hline Rastgoo et al. [48] & Vienna General Hospital & $\begin{array}{l}\text { Melanoma and } \\
\text { Dysplastic nev }\end{array}$ & $\begin{array}{l}\text { Sensitivity }-98 \% \\
\text { Specificity }-70 \%\end{array}$ \\
\hline Shimizu et al. [49] & $\begin{array}{l}\text { Keio University Hospital, } \\
\text { University of } \\
\text { Naples and Graz, } \\
\text { TokyoWomen'sMedical } \\
\text { University }\end{array}$ & $\begin{array}{l}\text { Melanoma, Nevus, } \\
\text { BCC, and SK }\end{array}$ & $\begin{array}{l}\text { Sensitivities-90.48\%, } \\
82.51 \%, \quad 82.61 \% \text { and } \\
80.61 \%\end{array}$ \\
\hline $\begin{array}{l}\text { Gonzàlez-Dìaz et } \\
\text { al. [50] }\end{array}$ & $\begin{array}{l}2017 \text { ISBI Challenge } \\
\text { database }\end{array}$ & $\begin{array}{l}\text { Melanoma, Nevus, } \\
\text { and SK }\end{array}$ & Sensitivity-95\% \\
\hline Esteva et al. [51] & $\begin{array}{l}\text { ISIC Dermoscopic } \\
\text { Archive, the } \\
\text { Edinburgh Dermofit } \\
\text { Library and data } \\
\text { from the Stanford Hospital }\end{array}$ & $\begin{array}{l}\text { Benign, malignant } \\
\text { and non-neoplastic }\end{array}$ & Sensitivity- $72.1 \%$ \\
\hline $\begin{array}{l}\text { Chatterjee et al. } \\
2019[39]\end{array}$ & $\begin{array}{l}\text { International Dermoscopic } \\
\text { Society } \\
\text { database, Dermoscopic } \\
\text { Atlas } \\
\text { database, ISIC: challenge } \\
\text { 2017, and } \\
\text { PH2 database }\end{array}$ & $\begin{array}{l}\text { Melanoma, Nevus, } \\
\text { BCC, and SK }\end{array}$ & $\begin{array}{l}\text { Sensitivities-98.76\%, } \\
99.01 \%, \quad 98.87 \% \text { and } \\
99.41 \%\end{array}$ \\
\hline $\begin{array}{l}\text { AI-Masni et al. } \\
{[38]}\end{array}$ & ISIC 2018 & $\begin{array}{l}\text { SK (AKIEC), } \\
\text { Melanoma, Nevus, } \\
\text { BCC, BKL, DF, } \\
\text { VASC }\end{array}$ & Accuracy $89.28 \%$ \\
\hline Proposed Model & ISIC 2018 & $\begin{array}{l}\text { SK (AKIEC), } \\
\text { Melanoma, Nevus, } \\
\text { BCC, BKL, DF, } \\
\text { VASC }\end{array}$ & $\begin{array}{l}\text { Accuracy } 91.7 \% \\
\text { Sensitivity }-100 \%, 100 \% \text {, } \\
66 \%, 100 \%, 63 \%, 79 \%, 100 \%\end{array}$ \\
\hline
\end{tabular}

It was observed in Table 8; the proposed methodology is the only method that deals with seven kinds of CPSL. Again, even if more kinds of CPSL are considered, the proposed method achieved better performance than the existing work. Also, the sensitivity of each class of CPSL is competent enough with the existing work.

The method achieved diagnostic effectiveness (accuracy) of $91.7 \%$, AUC is 0.99 , and LR+ is 49. As per STARD guidelines, the proposed diagnosis method is excellent (as AUC is within the range of 0.9 to 1 ) and an excellent diagnostic test (as LR+ > 10). In terms of definitely 
solving skin cancer issues for the classification of CPSL, the findings obtained signify that we are in the right direction.

\section{Compliance with ethical standards}

Funding: Any organization or institution does not support this research.

Conflict of interest: The authors declare that they have no conflict of interest.

Ethical approval: This article does not contain any studies with human participants or animals performed by any authors.

\section{Data availability statement:}

Data sharing does not apply to this article, as no new data were created or analyzed in this study.

\section{Reference}

[1] A. C. Society, "Cancer Facts \& Figures 2018," Atlanta, American Cancer Society, 2018.

[2] Koh HK. Melanoma screening: focusing the public health journey. Archives of dermatology. 2007 Jan 1;143(1):101-3

[3] Nikolaou V, Stratigos AJ. Emerging trends in the epidemiology of melanoma. British Journal of dermatology. 2014 Jan;170(1):11-9.

[4] A. C. Society, "Cancer Facts \& Figures 2008," Atlanta, American Cancer Society, 2008.

[5] Safigholi H, Meigooni AS, Song WY. Comparison of 192Ir, 169Yb, and 60Co highdose-rate brachytherapy sources for skin cancer treatment. Medical Physics. 2017 Sep;44(9):4426-36.

[6] Safigholi H, Song WY, Meigooni AS. Optimum radiation source for radiation therapy of skin cancer. Journal of applied clinical medical physics. 2015 Sep;16(5):219-27.

[7] Ouhib Z, Kasper M, Calatayud JP, Rodriguez S, Bhatnagar A, Pai S, Strasswimmer J. Aspects of dosimetry and clinical practice of skin brachytherapy: The American Brachytherapy Society working group report. Brachytherapy. 2015 Nov 1;14(6):84058.

[8] Dorj UO, Lee KK, Choi JY, Lee M. The skin cancer classification using deep convolutional neural network. Multimedia Tools and Applications. 2018 Apr 1;77(8):9909-24. 
[9] Esteva A, Kuprel B, Novoa RA, Ko J, Swetter SM, Blau HM, Thrun S. Dermatologist-level classification of skin cancer with deep neural networks. Nature. $2017 \mathrm{Feb} ; 542(7639): 115-8$.

[10] Ruiz D, Berenguer V, Soriano A, SáNchez B. A decision support system for the diagnosis of melanoma: A comparative approach. Expert Systems with Applications. 2011 Nov 1;38(12):15217-23.

[11] Mohan SV, Chang AL. Advanced basal cell carcinoma: epidemiology and therapeutic innovations. Current dermatology reports. 2014 Mar 1;3(1):40-5.

[12] Lindelöf B, Hedblad MA. Accuracy in the clinical diagnosis and pattern of malignant melanoma at a dermatological clinic. The Journal of dermatology. 1994 Jul;21(7):461-4.

[13] Morton CA, Mackie RM. Clinical accuracy of the diagnosis of cutaneous malignant melanoma. The British Journal of dermatology. 1998 Feb;138(2):283-7.

[14] Argenziano G, Soyer HP. Dermoscopy of pigmented skin lesions-a valuable tool for early. The lancet oncology. $2001 \mathrm{Jul}$ 1;2(7):443-9.

[15] Bafounta ML, Beauchet A, Aegerter P, Saiag P. Is dermoscopy (epiluminescence microscopy) useful for the diagnosis of melanoma? Results of a meta-analysis using techniques adapted to the evaluation of diagnostic tests. Archives of dermatology. 2001 Oct 1;137(10):1343-50.

[16] Vestergaard ME, Macaskill PH, Holt PE, Menzies SW. Dermoscopy compared with naked eye examination for the diagnosis of primary melanoma: a meta-analysis of studies performed in a clinical setting. British Journal of Dermatology. 2008 Sep;159(3):669-76.

[17] Salerni G, Terán T, Puig S, Malvehy J, Zalaudek I, Argenziano G, Kittler H. Meta-analysis of digital dermoscopy follow-up of melanocytic skin lesions: a study on behalf of the International Dermoscopy Society. Journal of the European Academy of Dermatology and Venereology. 2013 Jul;27(7):805-14.

[18] Binder M, Schwarz M, Winkler A, Steiner A, Kaider A, Wolff K, Pehamberger H. Epiluminescence microscopy: a useful tool for the diagnosis of pigmented skin lesions for formally trained dermatologists. Archives of dermatology. 1995 Mar 1;131(3):286-91.

[19] Braun RP, Rabinovitz HS, Oliviero M, Kopf AW, Saurat JH. Dermoscopy of pigmented skin lesions. Journal of the American Academy of Dermatology. 2005 Jan 1;52(1):109-21. 
[20] Kittler H, Pehamberger H, Wolff K, Binder MJ. Diagnostic accuracy of dermoscopy. The lancet oncology. 2002 Mar 1;3(3):159-65.

[21] Piccolo D, Ferrari A, Peris KE, Daidone R, Ruggeri B, Chimenti S. Dermoscopic diagnosis by a trained clinician vs. a clinician with minimal dermoscopy training vs. computer-aided diagnosis of 341 pigmented skin lesions: a comparative study. British Journal of Dermatology. 2002 Sep;147(3):481-6.

[22] Pehamberger H, Steiner A, Wolff K. In vivo epiluminescence microscopy of pigmented skin lesions. I. Pattern analysis of pigmented skin lesions. Journal of the American Academy of Dermatology. 1987 Oct 1;17(4):571-83.

[23] Steiner A, Pehamberger H, Wolff K. Improvement of the diagnostic accuracy in pigmented skin lesions by epiluminescent light microscopy. Anticancer research. 1987 May 1;7(3 Pt B):433-4.

[24] Dolianitis C, Kelly J, Wolfe R, Simpson P. Comparative performance of 4 dermoscopic algorithms by nonexperts for the diagnosis of melanocytic lesions. Archives of dermatology. 2005 Aug 1;141(8):1008-14.

[25] Carli P, Quercioli E, Sestini S, Stante M, Ricci L, Brunasso G, De Giorgi V. Pattern analysis, not simplified algorithms, is the most reliable method for teaching dermoscopy for melanoma diagnosis to residents in dermatology. British Journal of Dermatology. 2003 May;148(5):981-4.

[26] Burroni M, Corona R, Dell'Eva G, Sera F, Bono R, Puddu P, Perotti R, Nobile F, Andreassi L, Rubegni P. Melanoma computer-aided diagnosis: reliability and feasibility study. Clinical cancer research. 2004 Mar 15;10(6):1881-6.

[27] Gutman D et al., "Skin lesion analysis toward melanoma detection: A challenge at the international symposium on biomedical imaging (ISBI) 2016, hosted by the international skin imaging collaboration (ISIC)," 2016.

[28] Rosado B, Menzies S, Harbauer A, Pehamberger H, Wolff K, Binder M, Kittler H. Accuracy of computer diagnosis of melanoma: a quantitative meta-analysis. Archives of Dermatology. 2003 Mar 1;139(3):361-7.

[29] Masood A, Ali Al-Jumaily A. Computer-aided diagnostic support system for skin cancer: a review of techniques and algorithms. International Journal of biomedical imaging. 2013 Oct 30;2013.

[30] Barata C, Celebi ME, Marques JS. Improving dermoscopy image classification using color constancy. IEEE Journal of biomedical and health informatics. $2014 \mathrm{Jul}$ 25;19(3):1146-52. 
[31] Garnavi R, Aldeen M, Bailey J. Computer-aided diagnosis of melanoma using borderand wavelet-based texture analysis. IEEE Transactions on Information Technology in Biomedicine. 2012 Aug 8;16(6):1239-52.

[32] Glaister J, Wong A, Clausi DA. Segmentation of skin lesions from digital images using joint statistical texture distinctiveness. IEEE transactions on biomedical engineering. 2014 Jan 2;61(4):1220-30.

[33] Kaya S, Bayraktar M, Kockara S, Mete M, Halic T, Field HE, Wong HK. Abrupt skin lesion border cutoff measurement for malignancy detection in dermoscopy images. InBMC bioinformatics 2016 Oct 1 (Vol. 17, No. 13, p. 367). BioMed Central.

[34] Haroon M, Gallaghar P, Ahmad M, FitzGerald O. Elevated CRP even at the first visit to a rheumatologist is associated with long-term poor outcomes in patients with psoriatic arthritis. Clinical rheumatology. 2020 Apr 2.

[35] Chatterjee S, Dey D, Munshi S. Integration of morphological preprocessing and fractal-based feature extraction with recursive feature elimination for skin lesion types classification. Computer methods and programs in biomedicine. 2019 Sep 1; 178:20118.

[36] Birkenfeld JS, Tucker-Schwartz JM, Soenksen LR, Avilés-Izquierdo JA, Marti-Fuster B. Computer-aided classification of suspicious pigmented lesions using wide-field images. Computer Methods and Programs in Biomedicine. 2020 Oct 1; 195:105631.

[37] Balaji, V. R., S. T. Suganthi, R. Rajadevi, V. Krishna Kumar, B. Saravana Balaji, and Sanjeevi Pandiyan. (2020) Skin disease detection and segmentation using dynamic graph cut algorithm and classification through Naive Bayes Classifier. Measurement pp. 107922

[38] Al-Masni, M. A., Kim, D. H., \& Kim, T. S. (2020). Multiple skin lesions diagnostics via integrated deep convolutional networks for segmentation and classification. Computer Methods and Programs in Biomedicine, 190, 105351

[39] Chatterjee, Saptarshi, Debangshu Dey, Sugata Munshi, and Surajit Gorai. (2019) Extraction of features from cross-correlation in space and frequency domains for classification of skin lesions. Biomedical Signal Processing and Control 53, 101581

[40] Qin, Zhiwei, Zhao Liu, Ping Zhu, and Yongbo Xue. (2020) A GAN-based image synthesis method for skin lesion classification. Computer Methods and Programs in Biomedicine, pp.105568 
[41] Tschandl P, Rosendahl C, Kittler H. The HAM10000 dataset, a large collection of multi-source dermatoscopic images of common pigmented skin lesions. Scientific data. 2018 Aug 14; 5:180161

[42] Manohar N, Kumar YS, Rani R, Kumar GH. Convolutional Neural Network with SVM for Classification of Animal Images. In Emerging Research in Electronics, Computer Science and Technology 2019 (pp. 527-537). Springer, Singapore

[43] Agarap AF. An architecture combining convolutional neural network (CNN) and support vector machine (SVM) for image classification. arXiv preprint arXiv:1712.03541. 2017 Dec 10.

[44] Codella NC, Gutman D, Celebi ME, Helba B, Marchetti MA, Dusza SW, Kalloo A, Liopyris K, Mishra N, Kittler H, Halpern A. Skin lesion analysis toward melanoma detection: A challenge at the 2017 international symposium on biomedical imaging (isbi), hosted by the international skin imaging collaboration (ISIC). In2018 IEEE 15th International Symposium on Biomedical Imaging (ISBI 2018) 2018 Apr 4 (pp. 168-172). IEEE.

[45] Milton MA. Automated skin lesion classification using ensemble of deep neural networks in isic 2018: Skin lesion analysis towards melanoma detection challenge. arXiv preprint arXiv:1901.10802. 2019 Jan 30.

[46] Cohen JF, Korevaar DA, Altman DG, Bruns DE, Gatsonis CA, Hooft L, Irwig L, Levine D, Reitsma JB, de Vet HCW, Bossuyt PMM. STARD 2015 guidelines for reporting diagnostic accuracy studies: explanation and elaboration. BMJ Open 2016;6: e012799. http://bmjopen.bmj.com/content/6/11/e012799.abstract 
Figures

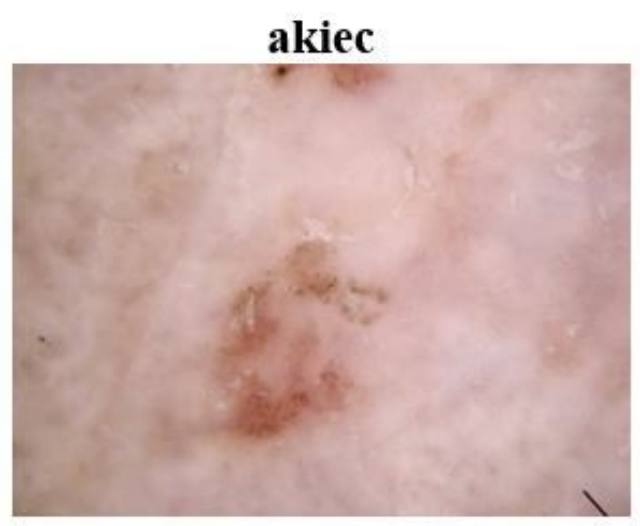

mel

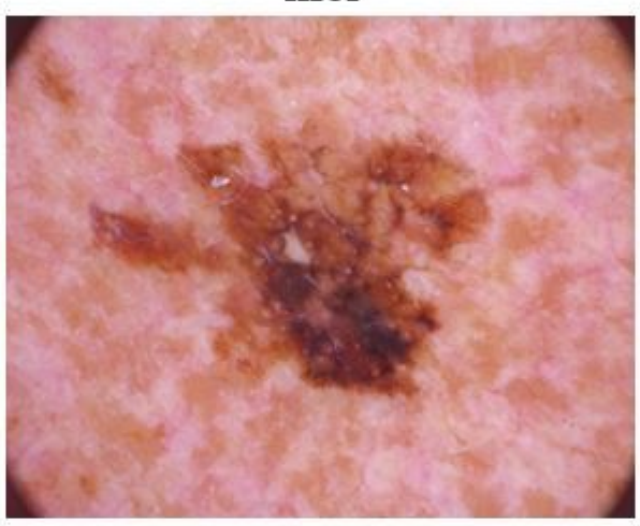

Vasc

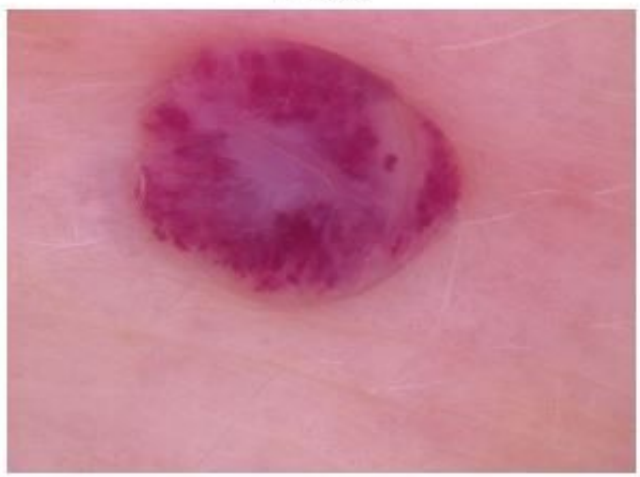

Figure 1

Sample images of CPSL

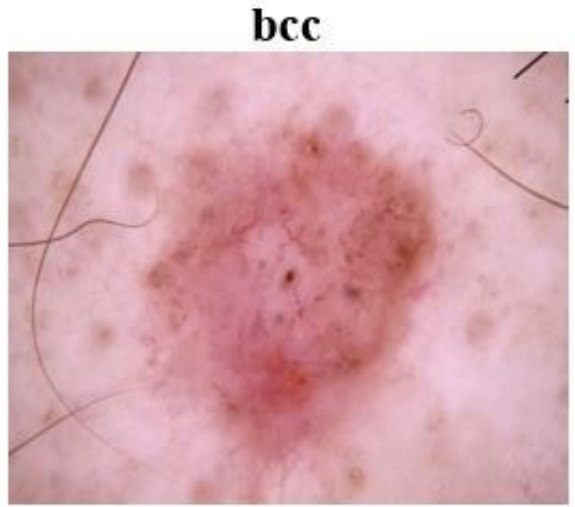

df

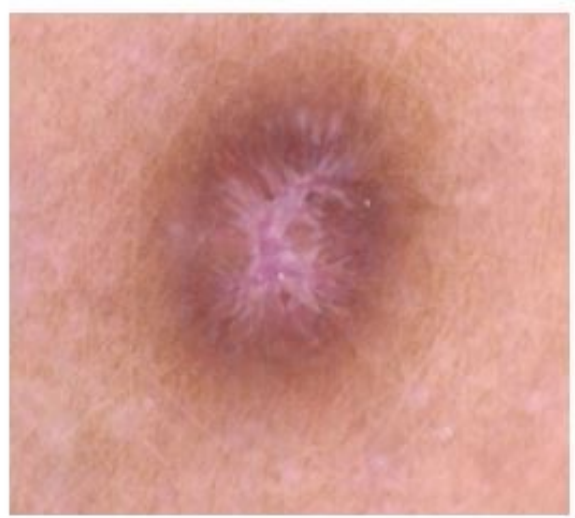

bkl

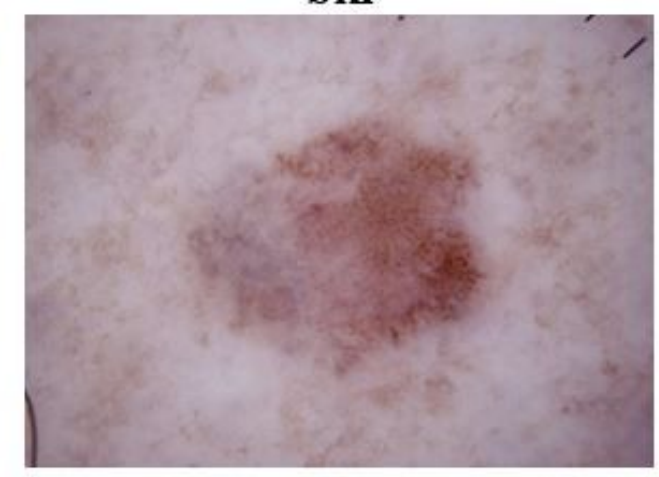

nV

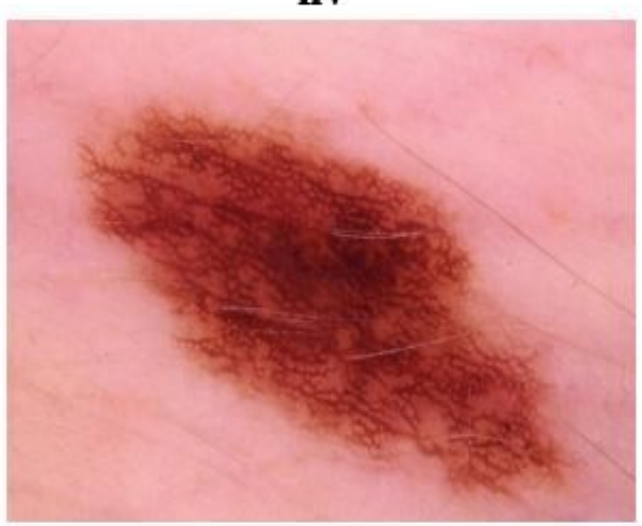




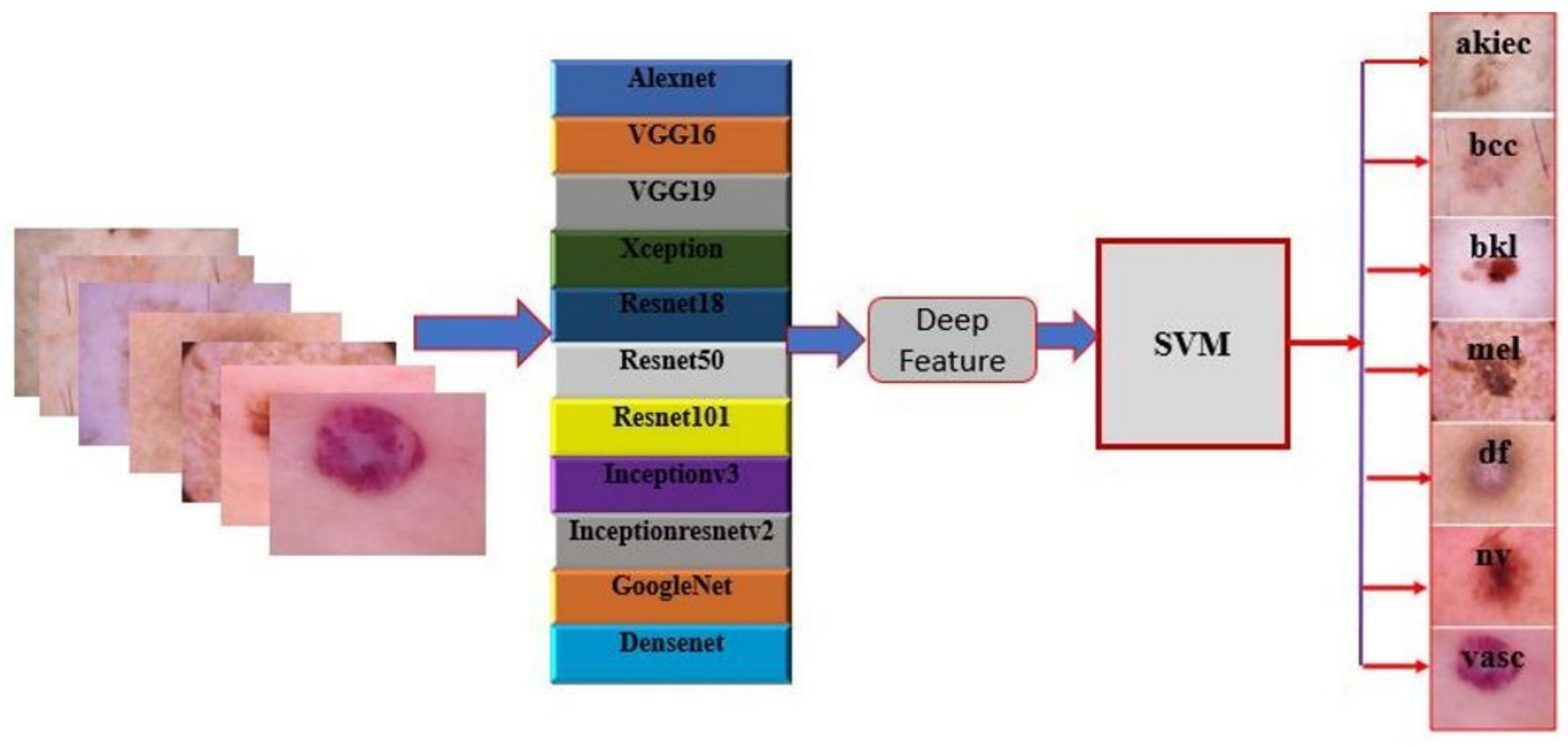

Figure 2

Performance Evaluation model of CNN for classification of CPSL

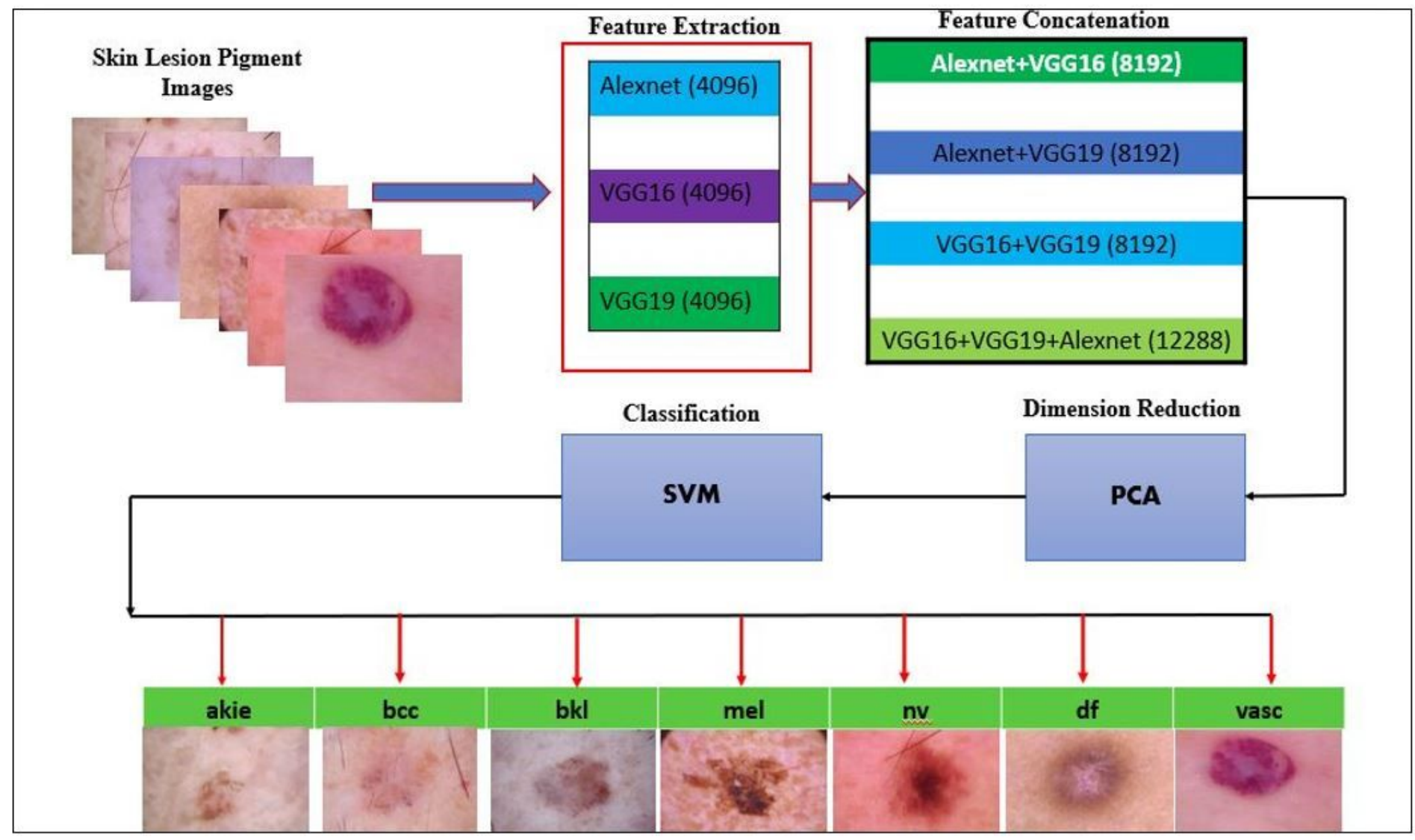

Figure 3 
The framework of the Proposed method based on multi-deep Features and SVM for the classification of CPSL

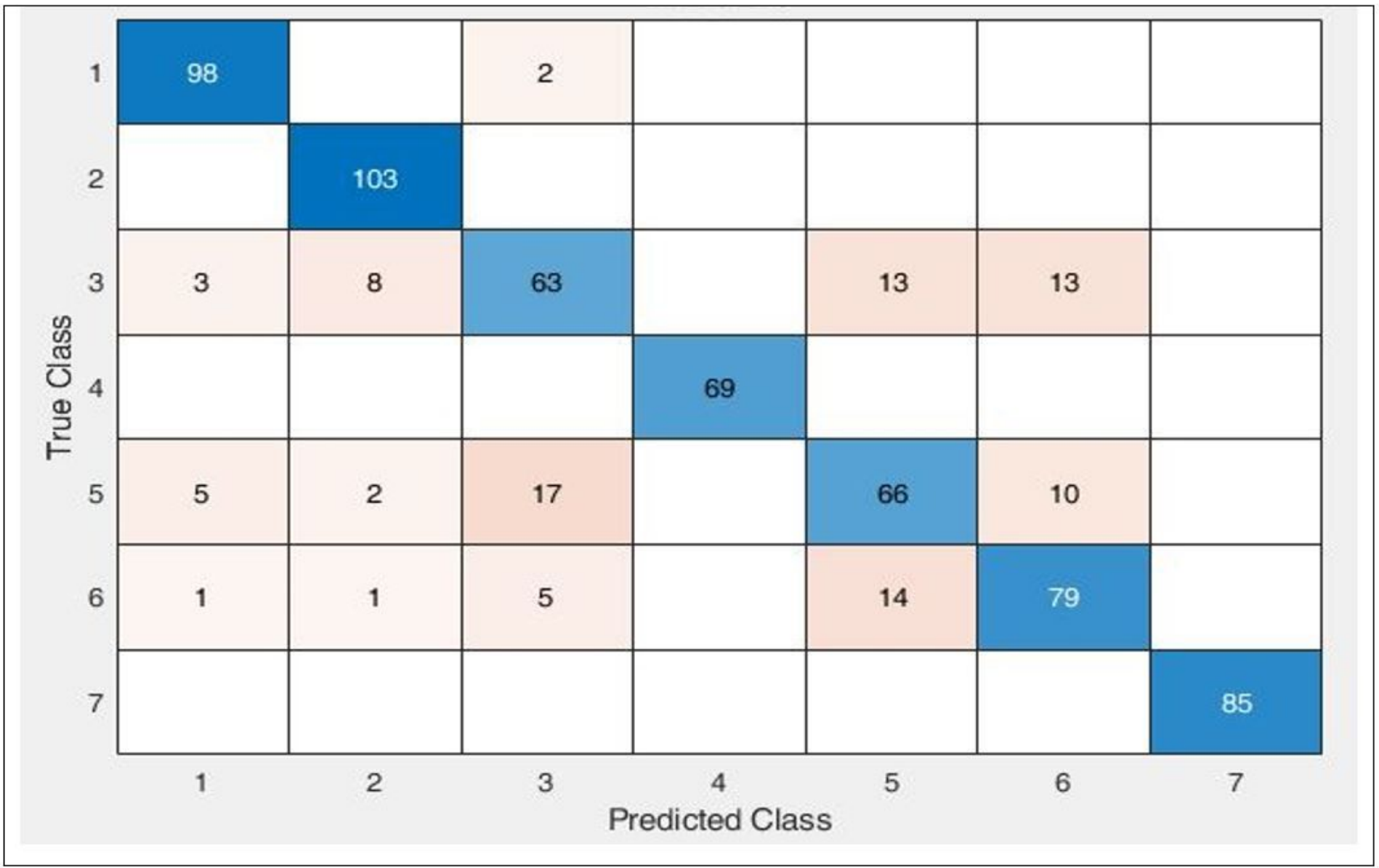

Figure 4

Confusion Matrix of Proposed classification model for diagnosis of breast carcinoma 


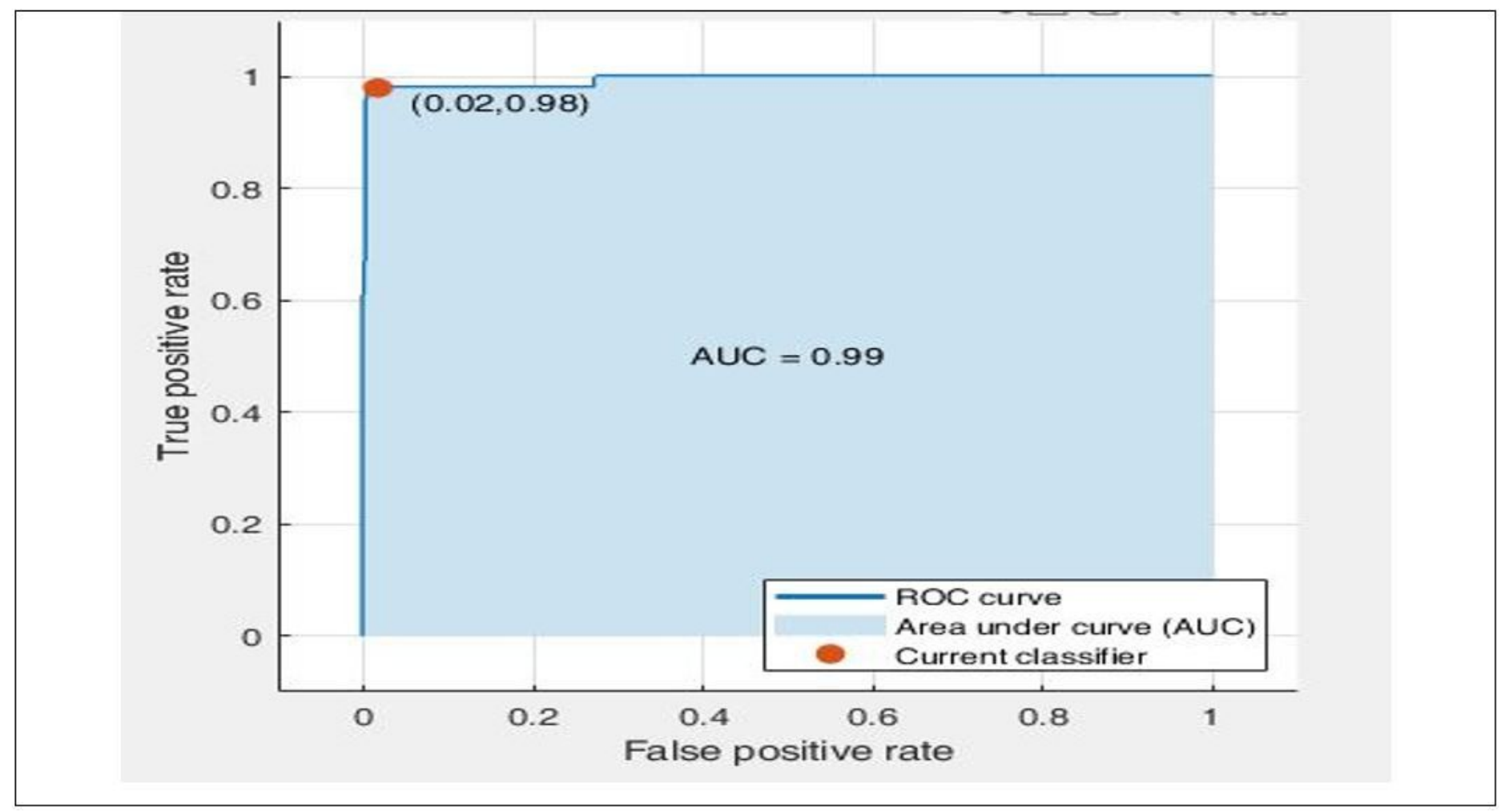

Figure 5

AUC of Proposed classification model for diagnosis of breast carcinoma 